\title{
Tape monitoring of the electrocardiogram in ambulant patients with sinoatrial disease
}

\author{
B. R. M. Crook, P. M. M. Cashman, F. D. Stott, and E. B. Raftery \\ From the Department of Cardiology, Northwick Park Hospital, and Division of Bioengineering, \\ Clinical Research Centre, Watford Road, Harrow, Middlesex
}

\begin{abstract}
Continuous tape recording of the electrocardiogram was carried out on 25 patients with sinus bradycardia or sinoatrial block. A 72-hour record was made on each fully ambulant patient, by a miniature portable tape recorder and the tapes processed with automatic dysrhythmia detection. A high incidence of slow and fast sinaatrial dysrhythmias was discovered and brief episodes of atrial tachydysrhythmias were especially common. The characteristic syndrome of sinoatrial dysrhythmias has been further clarified by this tape monitoring technique which has also proved to be valuable in the diagnosis and treatment of the individual patient.
\end{abstract}

A syndrome of sinus bradycardia, sinoatrial block, and fast atrial dysrhythmias occurring principally in middle-aged patients has been described under a variety of different names, e.g. 'The syndrome of alternating bradycardia and tachycardia' (Short, 1954), and 'The lazy sinus syndrome' (Eraut and Shaw, 1971), which emphasize selected aspects of this disorder. The aetiology of this syndrome is unknown, but it is thought to be due to abnormal function of the sinus node, and 'sinoatrial disease' is perhaps the most descriptive expression. The dysrhythmias are essentially intermittent (Bouvrain, Slama, and Temkine, 1967; Rubenstein et al., 1972), and their range and frequency can only be determined by long-term monitoring under ambulant conditions (Corday et al., 1965). We have examined a group of patients with this syndrome using a miniaturized tape recording system with an automatic recognition device in order to determine the type and frequency of their dysrhythmias.

\section{Patients and methods}

Twenty-five consecutive patients whose resting electrocardiograms had shown evidence of sinoatrial disease were selected from patients referred for cardiological advice. The criteria for selection were as follows. (I) 2nd or 3rd-degree sinoatrial block (Greenwood and Finkelstein, 1964); or (2) sinus bradycardia less than 56 beats per minute. All electrocardiograms were recorded for 3 days ( 72 hours) while the patients were fully ambulant at home and at work. A miniature analogue tape recorder (The Oxford Instrument Co. Ltd) was worn on a belt and 24-hour recordings were made on standard C 120 Received 2 April 1973. cassettes (Fig. I) (Littler et al., 1972). The electrocardiograph electrodes ('Gel Pad', Bionetics Inc for N.D.M. Corporation) were affixed to the chest to the right of the sternum in the second interspace and at the V6 position. This electrode system and placement was found to stay in position for the period of the recording and to give a good voltage QRS signal suitable for dysrhythmia analysis. The used tape cassette was replaced at the completion of each 24-hour recording period and the recorder batteries were renewed after each 48 hours.

The tapes were examined by a specially developed replay and analysis system (Fig. 2). Tape replay speed was $50 \mathrm{~mm} / \mathrm{sec}$ (or 25 times the recording speed) so that each 24-hour record was replayed in about I hour. With this speed of replay and with direct signal-plus-bias recording, the bandwidth available was approximately 0.2 to $100 \mathrm{~Hz}$. The analysis of dysrhythmias was based on $R R$ interval discrimination to provide recognition of premature beats, sinoatrial pauses, and slow or fast dysrhythmias. A shift register 'memory' was used to enable write-out of ro-second samples on a modified conventional electrocardiograph so that the dysrhythmic event appeared near the middle of the sample trace. The dysrhythmic event samples were then available for routine interpretation. In addition to the automatic dysrhythmia detection, a continuous visual display of the replayed electrocardiogram was in simultaneous use. On one oscilloscope, successive single QRS complexes were superimposed giving a constant image of QRS contour. On another, larger, oscilloscope, successive 30-second (real time) samples of the electrocardiogram were inscribed during each traverse of the beam. The visual display was not routinely observed but was surveyed during prolonged dysrhythmias or where automatic detection was sampling excessively as in the presence of interference.

The ro-second samples of the dysrhythmias were 


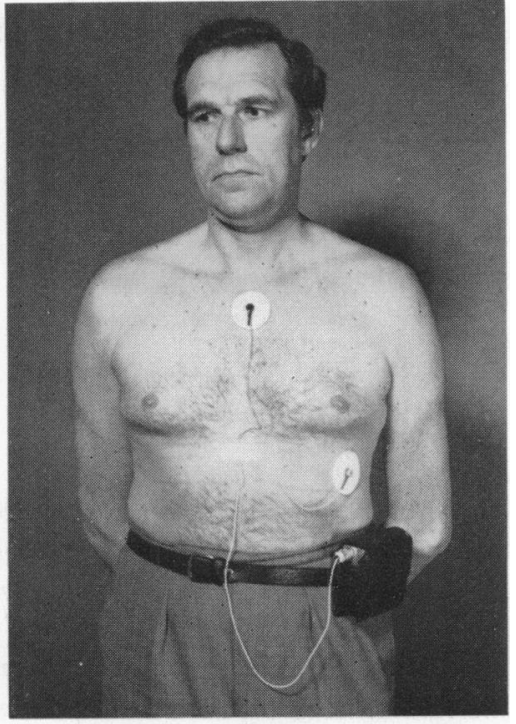

F I G. I Patient with electrodes in position and tape recorder attached to his belt. assessed and the range of dysrhythmias observed for each patient was noted and compared with the findings on the initial routine resting electrocardiogram.

\section{Results}

The group consisted of 15 men and 10 women, with a mean age of 62.6 years (range 23 to 79). A history of syncope was obtained in 9 patients, while 12 complained of 'dizzy turns' and II of 'palpitations'. In 9 patients there was electrocardiographic evidence of ischaemic heart disease ; 2 had a past history of diphtheria, and 2 had suffered rheumatic fever without evidence of valve involvement. Of the patients, I9 had a sinus bradycardia, with resting heart rates from 37 to 55 beats a minute, and II showed electrocardiographic evidence of sinoatrial block.

\section{a) Range of dysrhythmias observed}

Table I shows the incidence of the different dysrhythmias for each patient, both on routine resting electrocardiograms and on subsequent tape recording. Typical episodic dysrhythmias written out by

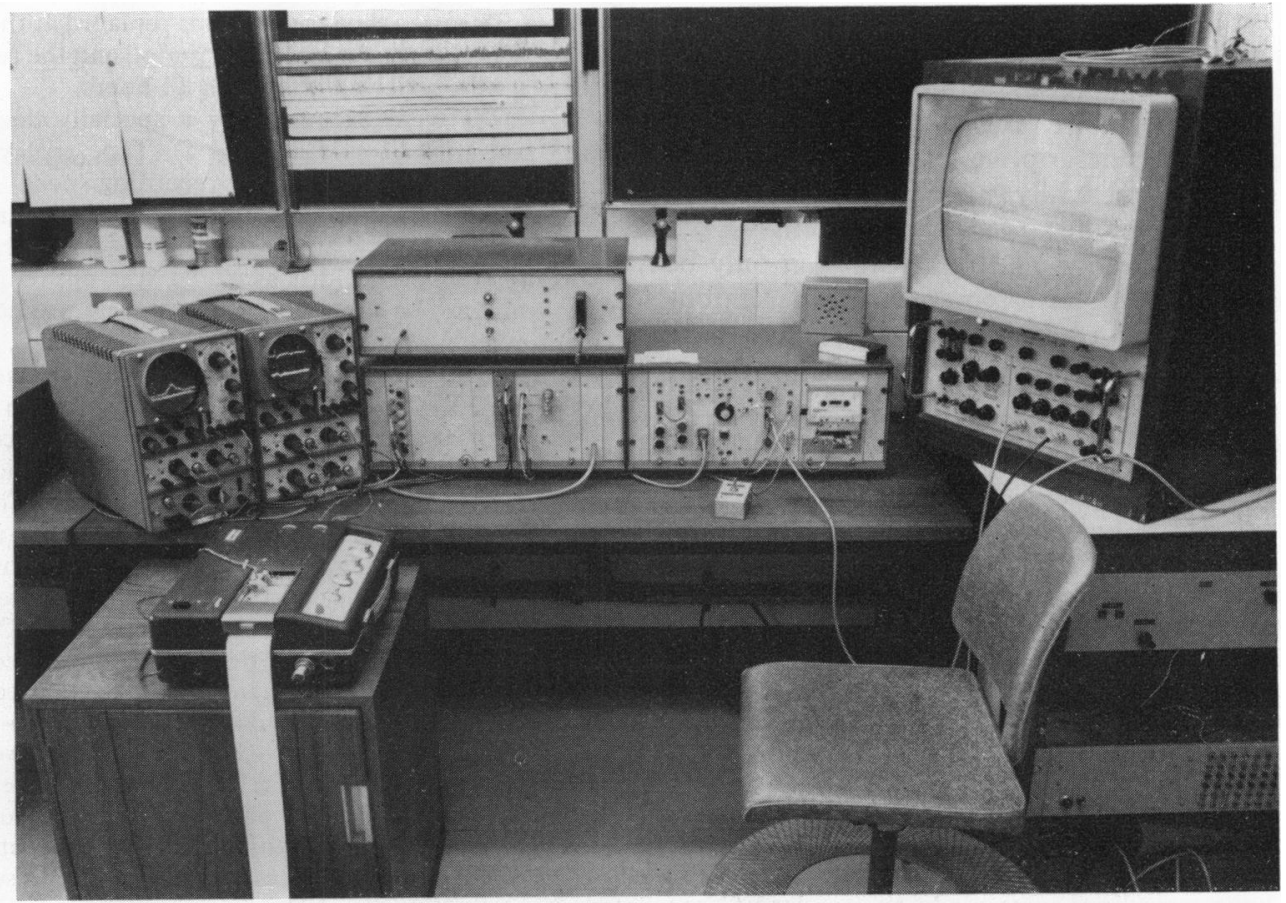

FIG. 2 Replay and analysis system. 
$\mathrm{TABLE} \mathrm{I}$ Incidence of dysrhythmias in 25 patients as found in routine resting electrocardiogram (shown as ' $O$ ') and as found on tape recordings (shown as ' $X$ ')

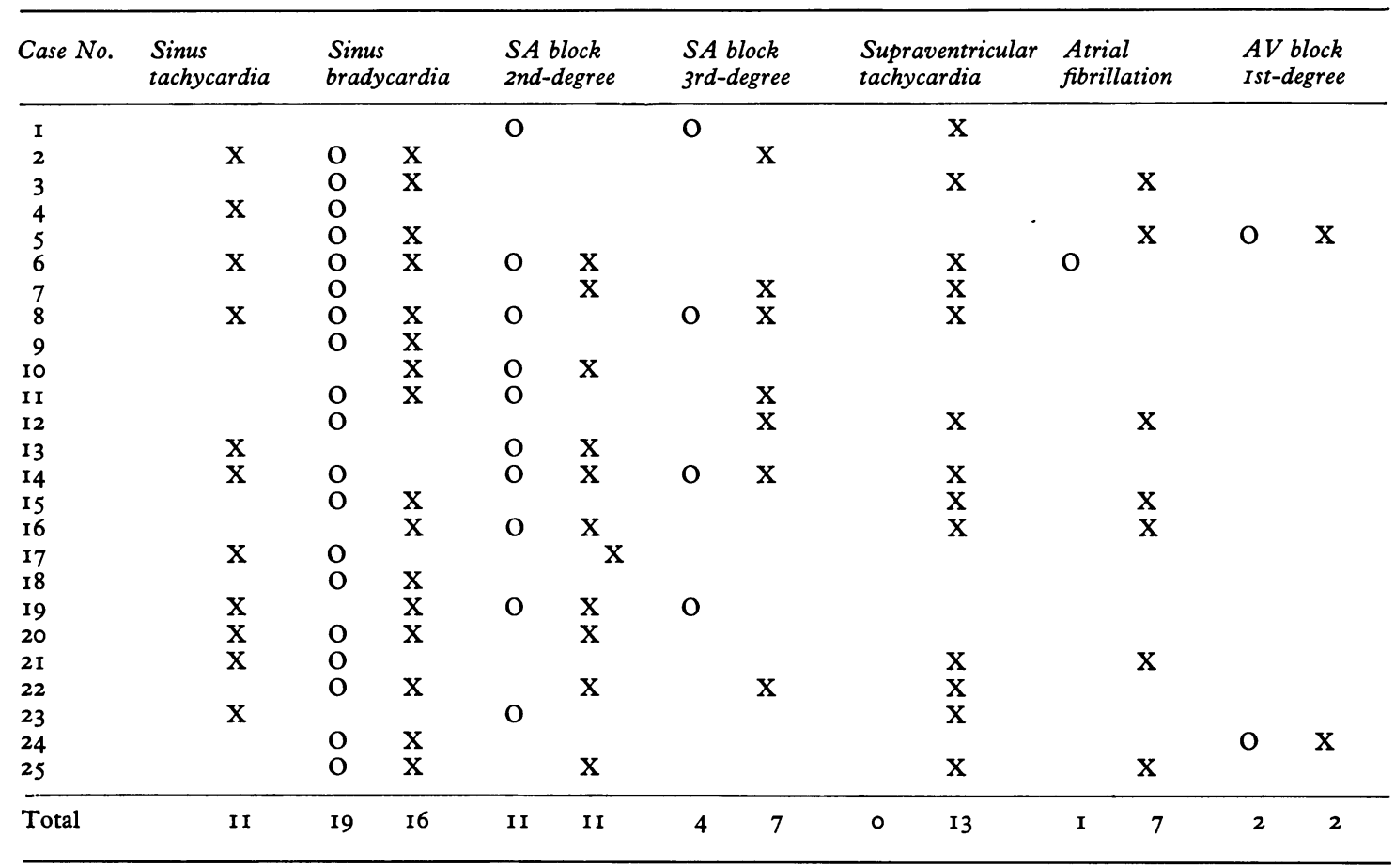

the automatic dysrhythmia detection system are seen in Fig. 3 and include junctional tachycardia, sinus bradycardia, and atrial fibrillation with sinoatrial block. In Table 2, a comparison is shown between the total incidence of the different dysrhythmias in all 25 patients (a) on routine resting electrocardiogram, (b) on tape record, and (c) on either (a) or (b). In Fig. 4 the same information is presented as a histogram. A high incidence of all the dysrhythmias was found on the tape records and especially atrial tachydysrhythmias which had been observed on the resting electrocardiogram in only one patient. The ventricular rate in the taperecorded episodes of supraventricular tachycardia ranged from 100 to 170 beats a minute, during atrial fibrillation from 120 to 150 beats a minute, and during sinus tachycardia from I 10 to 160 beats a minute. During the episodes of sinus bradycardia, the ventricular rate ranged from 36 to 56 beats a minute and the longest period of asystole from sinoatrial block was $3 \cdot 6$ seconds.

\section{b) Frequency and duration of dysrhythmic episodes}

The tachydysrhythmias were frequent, varying from I to about 30 in a 24-hour period and these episodes were usually brief, lasting for only a few seconds. One patient (Case 2r) was exceptional in having prolonged episodes of atrial fibrillation, each terminated by short asystolic pauses of about 2 seconds.

TABLE 2 Total incidence of each type of dysrhythmia in 25 patients, as detected by (a) routine resting electrocardiogram, (b) tape relay electrocardiogram, and $(c)$ either $(a)$ or $(b)$

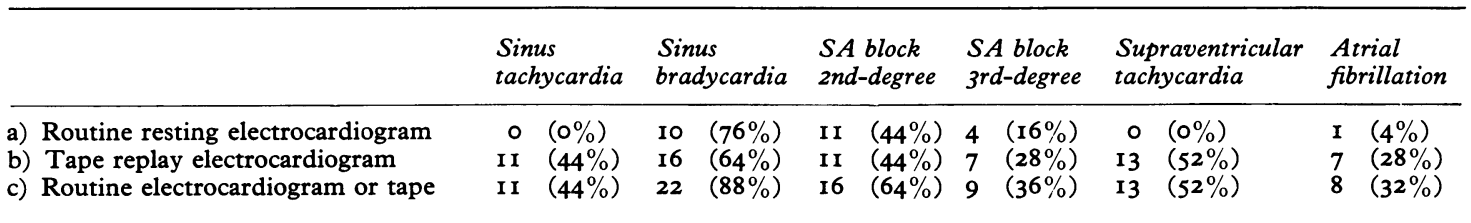


Junctional tachycardia

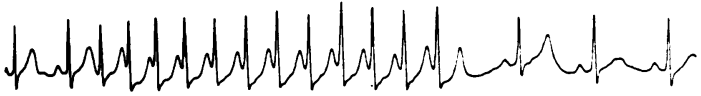

Sinus bradycardia

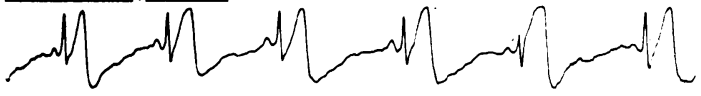

Atrial fibrillation with SA block

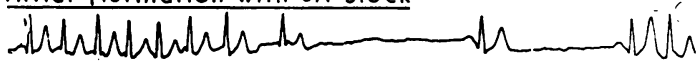

FIG. 3 Three dysrhythmic episodes from the tape recordings. Note the quality of the recordings and absence of muscle tremor or 'noise'.

\section{c) Technical problems and artefacts}

In general, satisfactory tape records and replay were achieved. Poor electrode contact or interference occurred occasionally, necessitating either adjustment of the dysrhythmia detection system to prevent excessive artefact sampling, or observation of the replay visual display and manual sampling. Two examples of technical problems are illustrated in Fig. 5. In the first electrocardiogram trace, interference yields a pattern which mimics supraventricular tachycardia. In the second, tape slip has occurred during recording giving the characteristic slurred PQRST patterns.

\section{Discussion}

The automatic system has proved effective in sampling intermittent dysrhythmias of all the categories

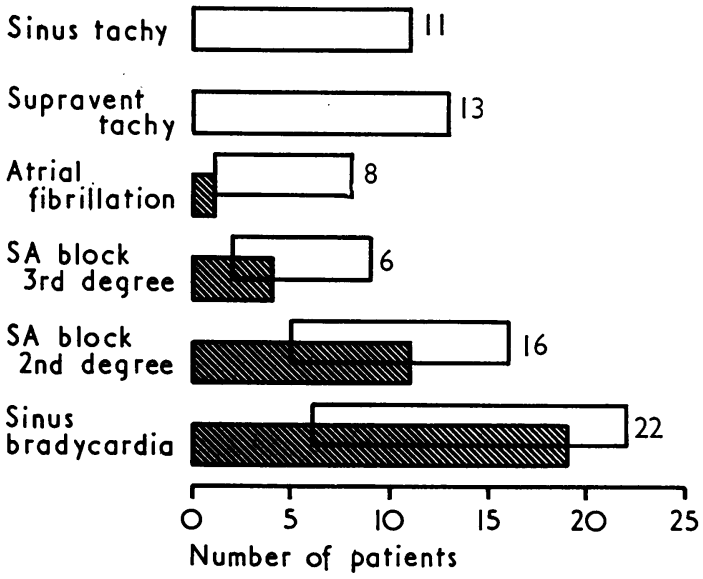

FIG. 4 Incidence of dysrhythmias detected on the resting electrocardiogram (hatched bars) and tape recorder (open bars). The figures at the ends of the bars are the total incidence from resting electrocardiograms and tape recordings.

usually found in sinoatrial disease, and study of the 'dynamic' electrocardiogram processed in this way has shown a high incidence of different dysrhythmias in the 25 patients. If sinus tachycardia is excluded, 20 of these 25 patients displayed at least one additional type of rhythm disorder on the tape record, which had not been found in the resting electrocardiogram (Table I). Of the 25 patients I4 had either atrial or junctional tachycardia, or atrial fibrillation, whereas only one of them had exhibited

STATIC INTERFERENCE

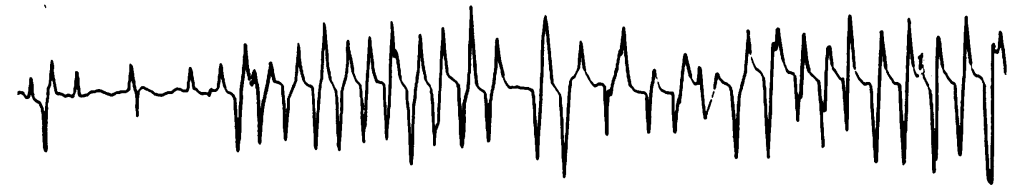

TAPE SLIP

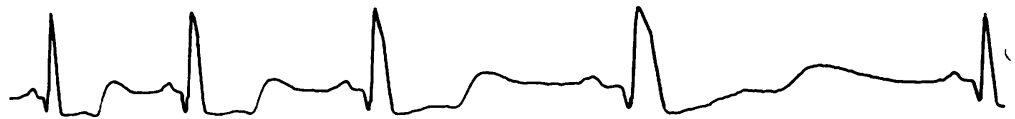

FIG. 5 Two common artefacts. The exact nature of static interference has not yet been established, but it is probably a consequence of friction between electrodes and clothes. The wide slurred QRST of tape slip is very characteristic. 
such a tachydysrhythmia on the routine resting electrocardiogram. In 2 patients with a history of syncopal attacks, short asystolic pauses of $2 \cdot 2$ and $3.6 \mathrm{sec}$ were recorded on tape but had not been found on the routine resting electrocardiogram.

It is also of interest to consider the 14 patients whose routine resting electrocardiogram showed sinus bradycardia. With tape recordings, ro of them exhibited either a fast dysrhythmia or sinoatrial block. It is therefore clear that individual patients commonly exhibited a wide range of dysrhythmias rather than any specific grouping such as 'brady-tachy' or a 'lazy sinus' with predominant sinus bradycardia.

This system of tape recording of the 'dynamic' electrocardiogram is clearly valuable as a means of diagnosing sinoatrial disease by confirming the characteristic rhythm disorders in the individual patient. Also, a knowledge of the nature and severity of the individual dysrhythmias should be helpful as an indication of the best method of treatment in this therapeutically challenging disorder. For example, two patients with short asystolic pauses and another with slow junctional rhythm have been greatly improved symptomatically by permanent pacing systems.

There must, however, be some reservation about the conclusions reached from this selected series of 25 patients, many of whom had symptoms and all of whom had been referred for cardiological advice. It would be of interest to know whether subjects with asymptomatic sinus bradycardia also exhibit a wide range of dysrhythmias, and this is at present under investigation. The criteria for the selection of these 25 patients were sinus bradycardia or sinoatrial block, as these dysrhythmias are more specific to sinoatrial disease than intermittent. fast atrial dysrhythmias which may, for example, be found with pre-excitation. It would, therefore, also be of interest to know if patients with unexplained fast atrial dysrhythmias have evidence of sinus bradycardia or sinoatrial block.

The sinus tachycardia (rate greater than IIO beats/minute) observed in II patients was unexpected. In 7 of them, rates from 130 to 160 beats a minute were observed. It is impossible to be certain whether these episodes were true sinus tachycardia or atrial tachydysrhythmias arising near the normal sinus pacemaker. Furthermore, 6 of 7 patients also showed sinus bradycardia. Since it has been shown that patients with sinus bradycardia have a poor response of heart rate to exercise, atropine, and isoprenaline (Eraut and Shaw, I97I; Crook et al., 1972) it appears unlikely that the observed sinus tachycardia is a normal physiological response. Possibly an ectopic pacemaker in or near the sinus node gives rise to this tachycardia.

In this study a comparable control group of normal subjects has not been investigated. There is, however, no indication from other dynamic electrocardiogram studies (Gilson, Holter, and Glasscock, 1964) that dysrhythmias of this kind occur in normal subjects, and in a preliminary study of ro normal subjects of comparable age to the patients with sinoatrial disease, no similar dysrhythmias were demonstrated on tape records.

\section{References}

Bouvrain, Y., Slama, R., and Temkine, J. (1967). Le bloc sino-auriculaire et les 'maladies du sinus'. Archives des Maladies du Coeur et des Vaisseaux, 60, 753.

Corday, E., Bazika, V., Lang, T. W., Pappelbaum, S., Gold, H., and Bernstein, H. (I965). Detection of phantom arrhythmias, and evanescent ECG abnormalities. Fournal of the American Medical Association, 193, 417.

Crook, B., Dighton, D., Leatham, A., and Siddons, H. (1972). Sino-atrial dysfunction presenting with bradycardia or sino-atrial block. In proceedings of the British Cardiac Society (abstract). British Heart fournal, 34, 962.

Eraut, D., and Shaw, D. B. (197I). Sinus bradycardia. British Heart fournal, 33, 742.

Gilson, J. S., Holter, N. J., and Glasscock, W. R. (1964). Clinical observations using the electrocardiorecorderAVSEP continuous electrocardiographic system. American fournal of Cardiology, 14, 204.

Greenwood, R., and Finkelstein, D. (1964). Sino-atrial Heart Block. (American lecture series.) Charles C. Thomas, Springfield, Illinois.

Littler, W. A., Honour, A. J., Sleight, P., and Stott, F. D. (1972). Continuous recording of direct arterial pressure and electrocardiogram in unrestricted man. British Medical fournal, 3, 76.

Rubenstein, J. J., Schulman, C. L., Yurchak, P. M., and DeSanctis, R. W. (1972). Clinical spectrum of the sick sinus syndrome. Circulation, 46, 5 .

Short, D. S. (1954). The syndrome of alternating bradycardia and tachycardia. British Heart fournal, 16, 208.

Requests for reprints to Dr. E. B. Raftery, Northwick Park Hospital, Watford Road, Harrow, Middlesex HAI 3 UJ. 